\title{
Monte Carlo Studies of Transverse Faraday Rotation Profiles
}

\author{
Eoin Murphy ${ }^{1, a}$ and Denise Gabuzda ${ }^{1, b}$ \\ ${ }^{1}$ Department of Physics, University College Cork, Ireland
}

\begin{abstract}
Faraday Rotation measurements are a very important tool for investigating the magnetic (B) fields associated with the relativistic jets of Active Galactic Nuclei (AGN); for example, a toroidal or helical B field component should give rise to a systematic gradient in the observed Faraday rotation across the jet. However, real observed radio images have finite resolution. Typical beam sizes for $\mathrm{cm}$-wavelength Very Long Baseline Interferometry (VLBI) observations are often comparable to or larger than the observed jet widths, raising questions about how well resolved a jet must be in the transverse direction in order to reliably detect transverse Faraday-rotation structure. The results of new simulations of Faraday rotation images designed to directly investigate this question are presented, together with studies of the probabilities of observing spurious Faraday Rotation gradients as a result of random noise and finite resolution. These simulations clearly demonstrate the possibility of detecting transverse Faraday-rotation structures even when the observed intrinsic jet widths are considerably smaller than the beam width.
\end{abstract}

\section{Introduction}

Faraday Rotation occurs when an electromagnetic wave propagates through a region with plasma and a magnetic field. The amount of rotation is given by

$$
\chi-\chi_{0}=R M \lambda^{2}
$$

where $\chi$ is the observed polarization angle, $\chi_{0}$ is the emitted polarization angle, $\lambda$ is the observing wavelength and $\mathrm{RM}$ is the Rotation Measure;

$$
R M=\frac{e^{3}}{8 \pi^{2} \epsilon_{0} m_{e}^{2} c^{3}} \int n_{e} \vec{B} \bullet \overrightarrow{d l}
$$

where $\mathrm{e}$ is the elementary charge, $\epsilon_{0}$ is the permittivity of free space, $m_{e}$ is the mass of the electron, $n_{e}$ is the number density of electrons in the plasma and $\mathrm{B}$ is the magnetic field strength in the plasma. We would expect to observe a transverse RM gradient if a helical $B$ field threads the jet, due to the systematic change in $\vec{B} \bullet \overrightarrow{d l}$ across the jet (Blandford 1993).

Faraday Rotation measurements are an important tool for investigating the $\mathrm{B}$ fields associated with the relativistic jets of Active Galactic Nuclei (AGN). However, real observed radio images have finite resolution. Typical beam sizes for cm-wavelength VLBI observations are comparable to or larger than the observed jet widths, raising questions about whether these jets are well enough resolved to reliably detect transverse Faraday-rotation structure (e.g. Taylor \& Zavala 2010).

\footnotetext{
a e-mail: eoin.g.murphy@gmail.com

be-mail: d.gabuzda@ucc.ie
}

This question has been investigated by Hovatta et al. (2012) and Algaba (2013), who used Monte Carlo simulations to consider the occurrence of spurious RM gradients due to noise and limited baseline coverage for various observed jet widths. In addition to carrying out such Monte Carlo simulations of our own, we have also considered the properties of observed RM gradients for various intrinsic jet widths relative to the beam width. Our new Monte Carlo simulations clearly demonstrate the possibility of detecting transverse Faraday-rotation structures even when the intrinsic jet widths are considerably smaller than the beam width (see also Mahmud et al. 2013).

\section{Monte Carlo Analysis Procedure}

We carried out Monte Carlo simulations based on a model source which has a transverse RM gradient across its jet. A simple, cylindrical model source which fell off in total intensity linearly with distance from the peak and the jet axis was used. This resulted in a "core-jet" like model with a jet length of about 20 mas (Fig. 1). A RM gradient was applied to the last three-quarters of the source length and the resultant Stokes $Q$ and $U$ maps generated.

The direct Fourier transform of these model maps at UV points based on existing snapshot observations with the Very Long Baseline Array were then calculated in order to produce model visibility data with realistic UV coverage. These data were generated for each of four wavelengths: either $18 \mathrm{~cm}, 20 \mathrm{~cm}, 21 \mathrm{~cm}$ and $22 \mathrm{~cm}$ (the wavelengths covered in the MOJAVE-I observations described by Coughlan et al. (2010)), or $1.9 \mathrm{~cm}, 2.3 \mathrm{~cm}$, 


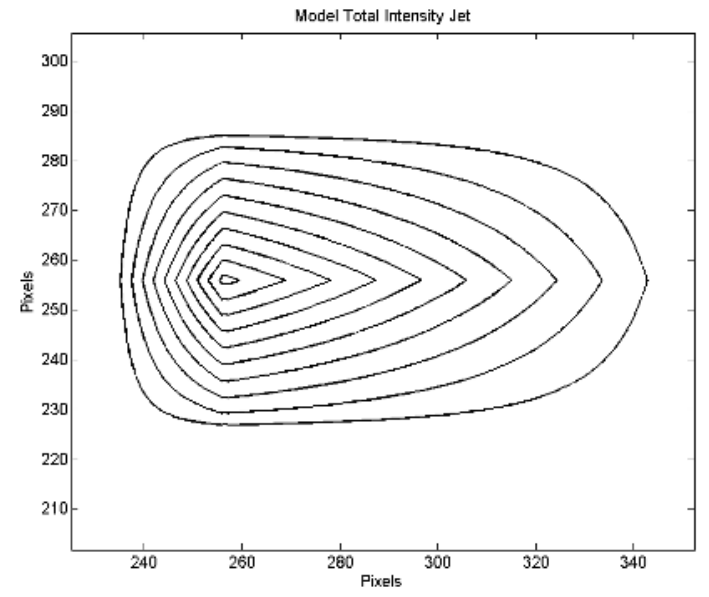

Figure 1: Sample total intensity image of the unconvolved model jet-like structure.

$3.4 \mathrm{~cm}$ and $3.8 \mathrm{~cm}$ (the wavelength range used by Hovatta et al (2012)). Random thermal noise and Electric Vector Position Angle (EVPA) calibration uncertainties of up to $3^{\circ}$ were added to these model visibilities.

The Stokes $Q$ and $U$ visibilites were then imaged in NRAO's CASA software using the natural-weight beam for the longest wavelength UV coverage. The $Q$ and $U$ images were then used to construct the corresponding polarization angle images at each frequency, which were, in turn, used to construct RM images in the usual way. Monte Carlo RM maps were constructed, based on 100 independent realizations of the thermal noise and EVPA calibration uncertainty, and an average RM map was derived by averaging together all 100 individual realizations of the RM distribution.

This entire procedure was repeated for model jets of different intrinsic jet widths, polarized fluxes and RM ranges. This allowed for direct analysis of the effect of finite resolution on observed RM profiles, in addition to the effects of fractional polarization and Faraday depolarization.

\section{Monte Carlo Results}

The analysis procedure outlined in Section 2 was completed for two different existing observations, a source at $+42^{\circ}$ declination observed at $18 \mathrm{~cm}, 20 \mathrm{~cm}, 21 \mathrm{~cm}$ and $22 \mathrm{~cm}$ and a source at $+32^{\circ}$ declination observed at $1.9 \mathrm{~cm}$, $2.3 \mathrm{~cm}, 3.4 \mathrm{~cm}$ and $3.8 \mathrm{~cm}$. Due to the different wavelength ranges, different RM gradients were applied to each. Examples of the Monte Carlo RM maps for the simulated $18-22 \mathrm{~cm}$ data can be seen in Figure 2.

Figure 3 shows the fraction of the Monte Carlo RM maps showing gradients whose differences on either side of the jet correspond to specified multiples of the uncertainty in the RM difference $\sigma$, ranging from $0 \sigma$ to $\geq 4 \sigma$, for two sets of the model sources investigated. Errors in the RM were calculated using the approach of Hovatta et al. (2012), assuming that the contribution of residual D-term errors is negligible, as is the case away from the peaks of images of sources that are not too bright. Our Monte Carlo results clearly demonstrate that RM gradients can be reliably detected for all the jet widths considered, including those yielding observed widths for the RM distribution comparable to a beam width - much narrow than the "three-beam-width" criterion of Taylor and Zavala (2010). For many of the jets with intrinsic widths of 0.20 and 0.40 beam widths, RM gradients with significances of $3 \sigma$ or greater were observed for the vast majority of their Monte Carlo realizations. Jets with intrinsic jet widths as small as 0.05 beam widths also demonstrated significant numbers of $3 \sigma$ gradients; for the relatively few cases for which this isn't true, the number of $2 \sigma$ gradients is much higher than is expected for purely spurious gradients, as is demonstrated in Section 4.

Additional MC simulations were undertaken to analyse the effects of both lower levels of polarized flux and smaller RM ranges on the visibility of RM gradients. In all cases, reducing the polarized flux reduced the fraction of observed gradients, with the narrower jets being more affected than wider jets by this change. However, high percentages of $3 \sigma$ gradients were observed for the widest intrinsic jet width in all but the most extreme cases. Reducing the RM range had very similar results to those found for reducing the polarized flux.

At first glance, it would seem that increasing the $\mathrm{RM}$ range covered by the transverse $\mathrm{RM}$ gradient should always make the gradient more visible. However, when the variation in polarization angle due to $\mathrm{RM}$ variations on scales much smaller than the convolving beam becomes too large, it leads to cancellation of the polarization in neighbouring regions (Faraday depolarization). Thus, it should be more difficult to detect transverse RM gradients across jets whose RM gradients are very strong.

\section{Spurious Gradients}

Utilizing the same procedure as that outlined in Section 2 but applying no RM Gradient to the simulated $Q$ and $U$ maps allowed direct investigation of the probability of observing spurious gradients, similar to the analyses done previously by Hovatta et al. (2012) and Algaba (2013). Simulated $Q$ and $U$ maps with a percentage polarization of $10 \%$ and a constant polarization angle were produced for jets with various intrinsic widths. Faraday RM maps were constructed for 1000 independent realizations of the thermal noise and EVPA calibration uncertainty, and transverse slices were taken across each of these realizations. Each slice was checked to see if the observed $\mathrm{RM}$ gradient along the slice was strictly monotonic. If it was, the difference between the RM values at the two ends of the slice was determined and its associated error calculated. Since no RM gradient had been applied to 

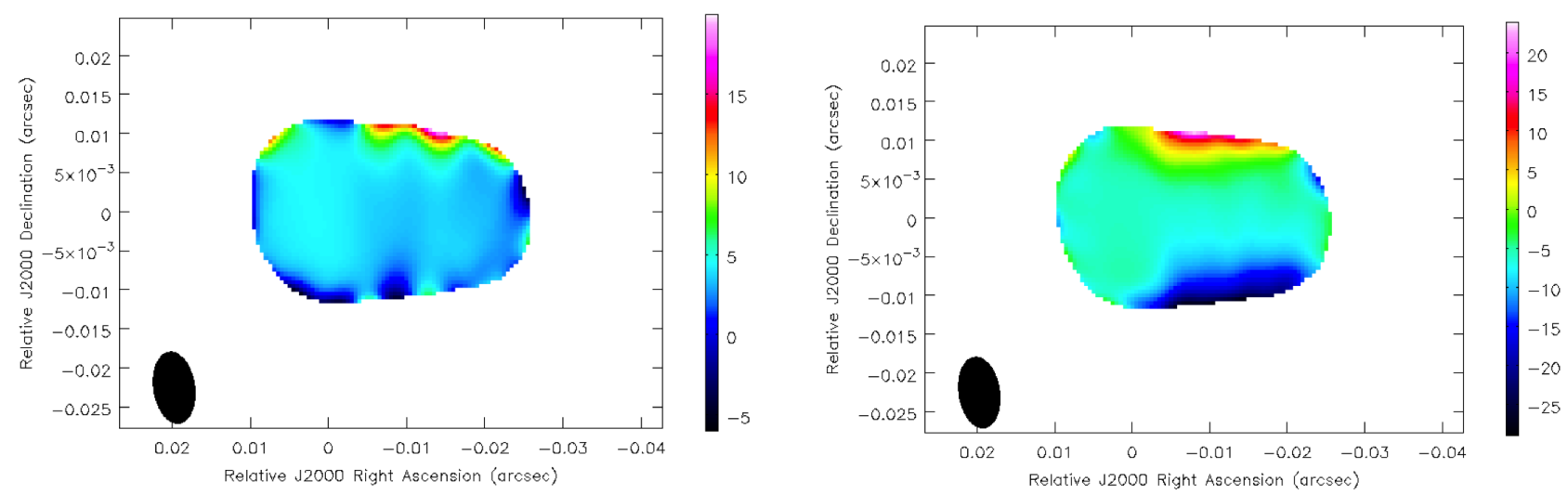

Figure 2: Sample Monte Carlo 18-22cm RM maps for model jets an intrinsic total transverse RM range $\pm 30 \mathrm{rad} \mathrm{m}^{-2}$ and with intrinsic jet widths of 0.05 beam widths (left) and 0.40 beam widths (right). While the gradient is less defined for the narrower jet width, it is still visible. The observed RM range is much closer to the intrinsic RM range for the wider jet.

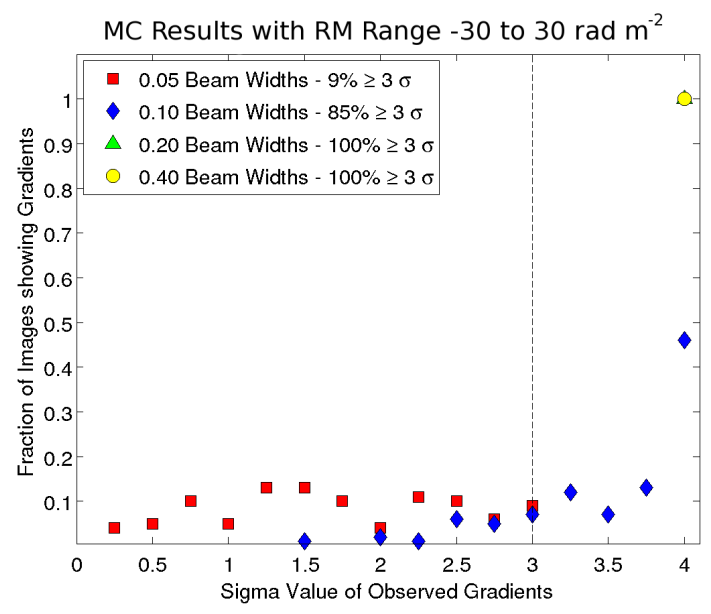

(a) MC Results for $18-22 \mathrm{~cm}$

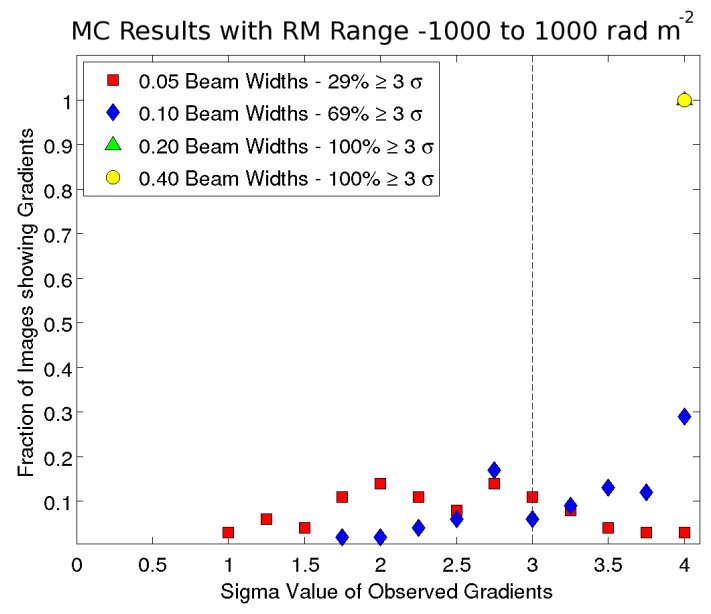

(b) MC Results for $1.9-3.8 \mathrm{~cm}$

Figure 3: Figures showing the fractions of MC Model Jet maps showing RM gradients. Note that the maximum Faraday rotation of the EVPAs is approximately equal in all cases. The left figures show results for $18-22 \mathrm{~cm}$ data and the right figures show results for $1.9-3.8 \mathrm{~cm}$ data.

the data, any monotonic RM gradients identified in this way were spurious, so that we were able to determine the probability of finding spurious gradients with various significances for various intrinsic jet widths.

Figure 4 shows the results of this analysis for the two wavelength ranges indicated in Section 2. As expected, the number of observed spurious gradients decreases as the observed jet width increases. The higher numbers of spurious gradients for the $1.9-3.8 \mathrm{~cm}$ simulations are due to the lower sensitivity of this limited wavelength range to Faraday rotation.

Spurious $4 \sigma$ gradients were very rarely observed. For the narrowest jet width, $4 \sigma$ gradients were observed in at most $\sim 0.5 \%$ of cases. No spurious $4 \sigma$ gradient were observed for observed jet widths of 1.5 beam widths. Spurious $3 \sigma$ gradients were also quite rare, and were observed in at most $\sim 1 \%$ of cases, even for the narrowest jet width ( 0.05 beam widths). In other words, the probability that observed monotonic RM gradients spanning values corresponding to $\geq 3 \sigma$ are spurious is no more than $1 \%$, for both the wavelength ranges considered here independent of the observed width of the RM gradient.

Spurious $2 \sigma$ were observed in at most $\sim 12 \%$ of cases. Inspection of Fig. 3 showing our results for simulated RM gradients indicates that the fraction of images showing gradients at the $2 \sigma$ level can appreciably exceed the fraction of spurious $2 \sigma$ gradients. Therefore, the possible 

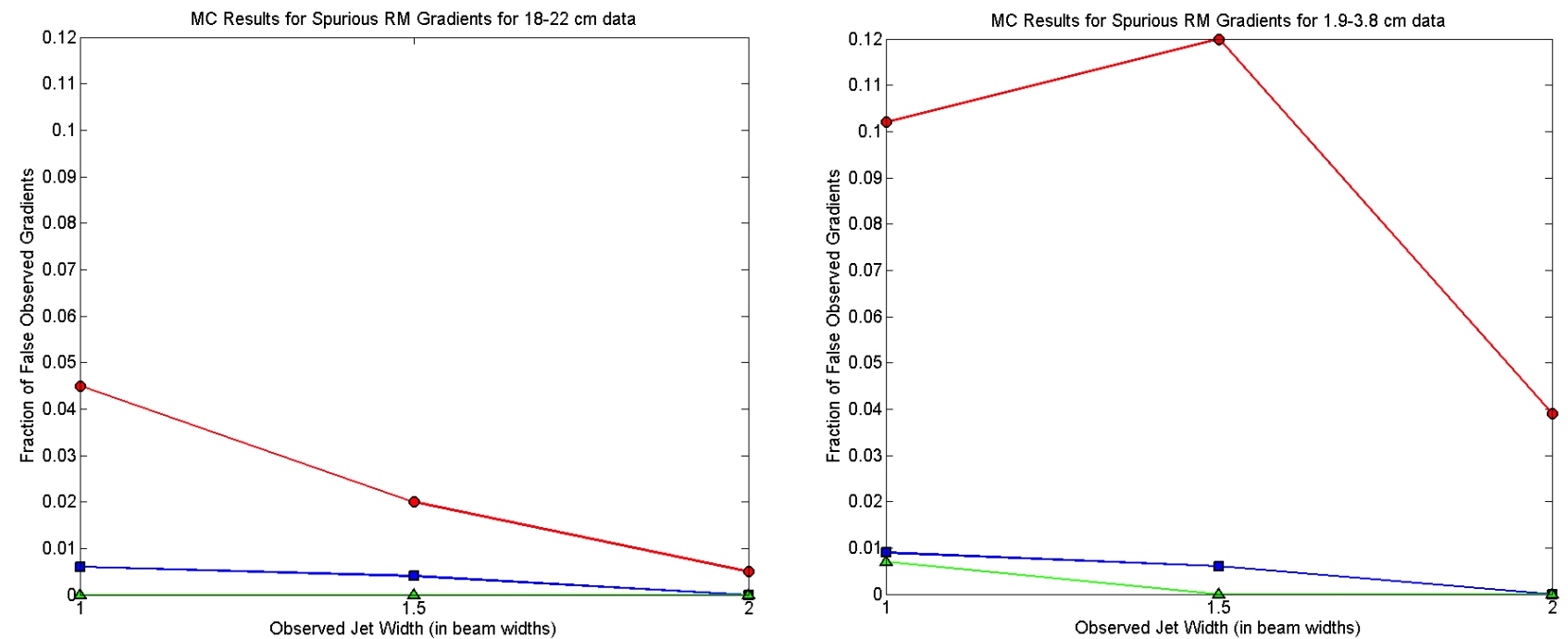

Figure 4: The above figures show the fraction of spurious monotonic gradients observed fr the $18-22 \mathrm{~cm}$ (left) and $1.9-3.8 \mathrm{~cm}$ (right) simulations ( $2 \sigma$ as red circles, $3 \sigma$ as blue squares and $4 \sigma$ as green triangles). $3 \sigma$ or greater spurious gradients were observed in fewer than $1 \%$ of cases at both wavelength ranges, even for the narrowest model jets considered.

reality of an observed $2 \sigma \mathrm{RM}$ gradient should not be completely ruled out, and such gradients should be treated as tentative results that must be confirmed by further data, as was also pointed out by Hovatta et al. (2012).

\section{Conclusions}

Taylor and Zavala (2010) proposed that an observed width of at least 3 beam widths should be required in order for a transverse RM gradient to be reliable. We have demonstrated that this criteria is far too severe: it may be possible to detect transverse RM gradients even when the intrinsic jet width is only $0.05-0.10$ of the beam width, depending on the wavelength range used. The reliability of observed RM gradients should instead be estimated from the RM difference across the jet $(>3 \sigma)$, the monotonicity of the gradient, the extent of the RM gradient along the jet, the quality of the RM fits and the possibility of distortion by optical depth effects.

Spurious RM gradients at the $3 \sigma$ level occurred in less than $1 \%$ of our simulations using model data without RM gradients, even in the case of very narrow jets (down to 0.01 of the beam width for the $18-22 \mathrm{~cm}$ simulations!). Thus, in the absence of other grounds for doubt (e.g. poor RM fits), monotonic transverse RM gradients spanning $\mathrm{RM}$ differences of $3 \sigma$ or more can be considered reliable, even if the transverse distance spanned is comparable to the beam width.

\section{Acknowledgements}

Funding for this research was provided by the Irish Research Council for Science Engineering and Technology (IRCSET).

\section{References}

[1] Blandford R. D. 1993, Astrophysical Jets, Ed. D. Burgarella, M. Livio, C. O’Dea, Cambridge University Press, p. 15

[2] Taylor, G.B., Zavala, R., ApJ L183, 722 (2010)

[3] Hovatta, T., Lister, M.L., Aller, M.F., Homan, D.C., Kovalev, Y.Y., Pushkarev, A.B., Savolainen,T., ApJ 144, 105 (2012)

[4] Algaba J.C., MNRAS 429, 3551 (2013)

[5] Mahmud, M., Coughlan C., Murphy E., Gabuzda, D.C.; Hallahan, D.R., MNRAS 431, 695 (2013)

[6] Coughlan, C.; Murphy, R.; McEnery, K.; Patrick, H.; Hallahan, R.; Gabuzda, D., Proceedings of the 10th European VLBI Network Symposium. Published online at http://pos.sissa.it/cgi-bin/reader/conf.cgi?confid=125, id.46 (2010) 